\title{
BLASTOMICOSIS ORAL, PAPILOMATOSIS LARÍNGEA Y TUBERCULOSIS ESOFÁGICA
}

\author{
Manuel Montoya ${ }^{1,2, a}$, Robert Chumbiraico ${ }^{1,2, b}$, Melvin Ricalde ${ }^{1,2, c}$, Ernesto Cazorla ${ }^{1,2, d}$, \\ Gustavo Hernández-Córdova ${ }^{2,3, e}$
}

\begin{abstract}
RESUMEN
El compromiso esofágico es una complicación infrecuente de la tuberculosis incluso en países con alta prevalencia de infección. Se presenta el caso de un paciente de 57 años no seropositivo al virus de inmunodeficiencia humana $(\mathrm{VIH})$, con diagnóstico simultáneo de blastomicosis en cavidad oral y papilomatosis laríngea, ambas confirmadas por anatomía patológica. La biopsia de esófago reveló esofagitis granulomatosa con necrosis; la tinción de Ziehl-Neelsen mostró bacilos ácido-alcohol resistente, sugerentes de tuberculosis. El antecedente de tuberculosis pulmonar en dos oportunidades y abandono de tratamiento determinó el inicio de tratamiento antituberculoso de segunda línea a través de un tubo de gastrostomía, más itraconazol vía oral. La evolución fue favorable.
\end{abstract}

Palabras clave: Blastomicosis; Tuberculosis; Esofagopatías; Papiloma; Micosis (fuente: DeCS BIREME).

\section{ORAL BLASTOMYCOSIS, LARYNGEAL PAPILLOMATOSIS AND ESOPHAGEAL TUBERCULOSIS}

\begin{abstract}
Esophageal involvement is an extremely rare complication of tuberculosis even in countries with high prevalence of infection. We report the case of a 57 year-old hiv-seronegative patient with simultaneous diagnoses of oral blastomycosis and laryngeal papillomatosis. Both were confirmed by anatomopathological analysis. The esophageal biopsy revealed granulomatous esophagitis with necrosis and ziehl-neelsen stain showed acid-fast alcohol resistant bacilli suggestive of tuberculosis. The patient's history included pulmonary tuberculosis twice and previous abandonment of therapy. Thus, it was necessary to use oral itraconazole combined with second-line anti-tuberculosis drugs administered through a gastrostomy tube. The clinical development was favorable.
\end{abstract}

Keywords: Blastomycosis; Tuberculosis; Esophageal diseases; Papilloma; Mycoses (source: MeSH NLM).

\section{INTRODUCCIÓN}

El compromiso esofágico es una complicación infrecuente de la tuberculosis (TB). Presenta una prevalencia de alrededor del $0,15 \%$ de los casos de TB y $0,3 \%$ con relación a los casos específicos de TB gastrointestinal ${ }^{(1,2)}$. Generalmente se ve afectado el tercio medio esofágico a la altura de la carina ${ }^{(1,3)}$, y los síntomas se presentan según el tipo de compromiso: disfagia (en el $90 \%$ de casos) en la forma hipertrófica, y dolor retroesternal con odinofagia en la forma ulcerativa ${ }^{(2,4)}$. Se debe considerar el diagnóstico diferencial con carcinoma esofágico ${ }^{(1,3)}$.

Existen varios mecanismos de infección: inoculación por esputo deglutido; extensión directa a partir de una tuberculosis faríngea; diseminación hemática de un sitio distante, o diseminación linfática retrógrada desde las glándulas peritraqueales y peribronquiales (asiento frecuente de TB). La mayoría de los casos ocurre desde estructuras adyacentes como ganglios linfáticos mediastinales, focos pulmonares, o infección vertebral tuberculosa en la región cervical ${ }^{(1,2)}$.

El diagnóstico de TB esofágica se basa en el hallazgo de granulomas en la biopsia esofágica en la que se ha descartado otros agentes etiológicos y, fundamentalmente, por el crecimiento de Mycobacterium tuberculosis en el cultivo ${ }^{(5)}$. La tomografía o la ecoendoscopía (aún poco utilizadas para el diagnóstico inicial) permiten diferenciar la TB esofágica primaria de la secundaria ${ }^{(1)}$.

El Paracoccidioides brasiliensis es el agente causal de la paracoccidioidomicosis o blastomicosis sudamericana; infección crónica (especialmente en adultos) y

\footnotetext{
Hospital Regional del Cusco. Cusco, Perú.

Universidad Nacional de San Antonio Abad del Cusco. Cusco, Perú.

Sociedad Científica de Estudiantes de Medicina ASOCIEMH-CUSCO. Cusco, Perú.

Médico infectólogo; ${ }^{\mathrm{b}}$ médico internista; ${ }^{\mathrm{c}}$ médico neumólogo; ${ }^{\mathrm{d}}$ médico gastroenterólogo; ${ }^{\mathrm{e}}$ estudiante de Medicina
} 
granulomatosa cuya diseminación hemática origina una amplia gama de manifestaciones en la piel, mucosas, ganglios y vísceras. Este hongo se adquiere por inhalación, se encuentra en el suelo y materia vegetal en su fase micelial porógena, y como levadura en tejidos infectados. Afecta mayoritariamente a hombres (90\%) entre los 30 y 50 años ${ }^{(6,7)}$. En el Perú, la blastomicosis se ha encontrado con mayor frecuencia en su forma clínica mucocutánea de inicio bucal ${ }^{(8)}$.

La papilomatosis laríngea es una enfermedad infrecuente producida por el virus papiloma humano que causa obstrucción de las vías respiratorias y alteraciones en la voz por su facilidad de causar infección en la tráquea y laringe. Es recurrente aun después de resección quirúrgica con riesgo de malignización ${ }^{(9)}$.

\section{REPORTE DE CASO}

Paciente varón de 57 años, procedente de la ciudad de Quillabamba (selva baja del Perú), obrero de construcción civil. Presenta el antecedente de haber tenido TB pulmonar dos años antes del ingreso, con tratamiento incompleto por abandono a los cuatro meses del esquema I. Un año después siguió tratamiento por ocho meses para TB pulmonar bajo el esquema II.

El cuadro clínico actual se inició con odinofagia aproximadamente un año antes del ingreso. El paciente lo relacionó con la aparición de lesiones dolorosas en la lengua. Seis meses después se agregó disfagia progresiva con incapacidad para deglutir, primero a sólidos y luego a líquidos, así como tos con expectoración blanquecina, disfonía, fiebre, dolor abdominal, hiporexia, cefalea y disminución marcada de peso.

Al examen físico, se vio un halo excoriativo dorso nasal (en la rinoscopia); lengua hipotrófica con lesiones

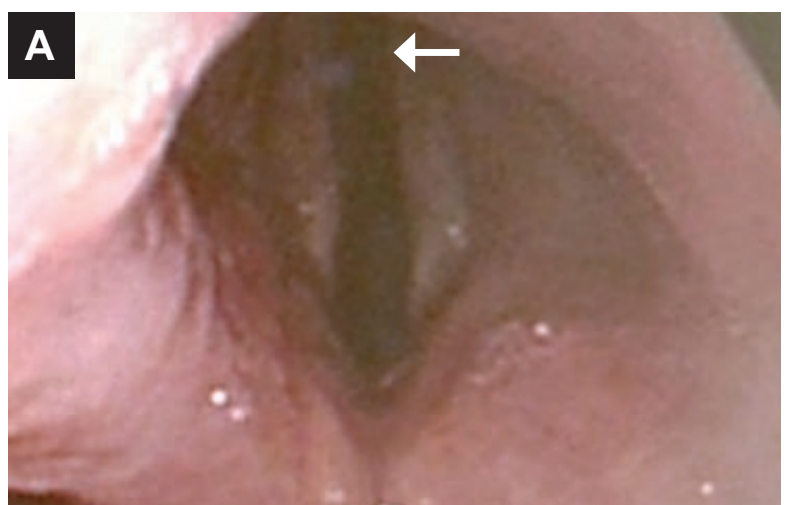

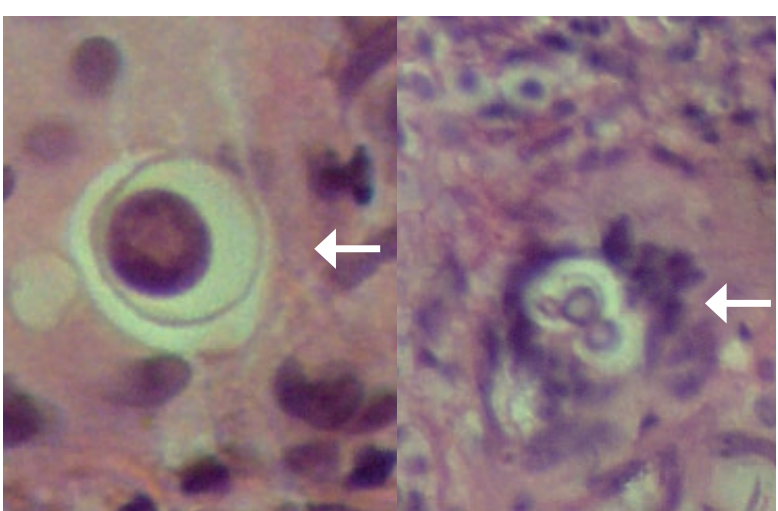

Figura 1. Inflamación crónica granulomatosa provocada por Paracoccidioides brasiliensis (coloración HE 400x).

blanquecinas hiperqueratósicas con fondo eritematoso, dolorosas en los lados y el dorso. Vibraciones vocales y murmullo vesicular sin alteraciones en ambos hemitórax. Evaluación cardiovascular sin alteraciones.

Los hallazgos de laboratorio a la semana del ingreso, revelaron anemia (hematocrito: 32\%, hemoglobina: $10,8 \mathrm{~g} / \mathrm{dL})$. El hemograma mostró leucocitos en $11300 \mathrm{cel} / \mathrm{mm}^{3}$, con $72 \%$ de neutrófilos; $3 \%$ de abastonados; $13 \%$ de linfocitos; $6 \%$ de monocitos; $9 \%$ de eosinófilos, y $0,1 \%$ de basófilos. El análisis bioquímico sanguíneo mostró: glucosa, $84 \mathrm{mg} / \mathrm{dL}$; urea, $49 \mathrm{mg} / \mathrm{dL}$; creatinina, 2,73 mg/dL; fosfatasa alcalina; $487 \mathrm{U} / \mathrm{L}$, albúmina, 3,5 g/dL; bilirrubina total, $0,30 \mathrm{mg} / \mathrm{dL}$; alaninoaminotransferasa (ALT), 29U/L; aspartatoaminotransferasa (AST), 15U/L; tiempo de protrombina, $17,8 \mathrm{~s}$; e INR 1,60. Preocupó los valores séricos de calcio y fósforo $(7,0$ y $11,8 \mathrm{mg} / \mathrm{dL}$ respectivamente). El valor de adenosindeaminasa sérica (ADA) fue de 15,4 U/L.

En la lámina periférica se observó anisocitosis en la serie eritrocítica, predominio de neutrófilos maduros y polimorfonucleares con granulaciones tóxicas. La

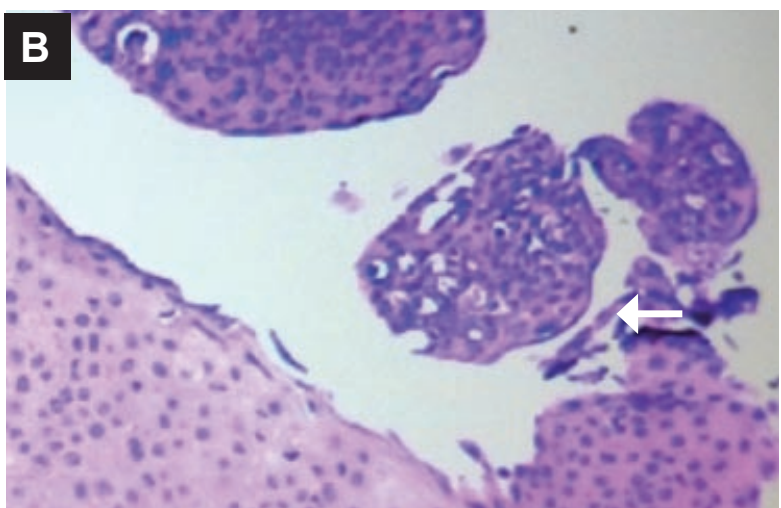

Figura 2. Papilomatosis laríngea. A. Inspección endoscópica del daño en las cuerdas vocales; B. Papiloma, lesión con cambios citopáticos sugestivos de infección por virus papiloma humano (coloración HE 100x). 

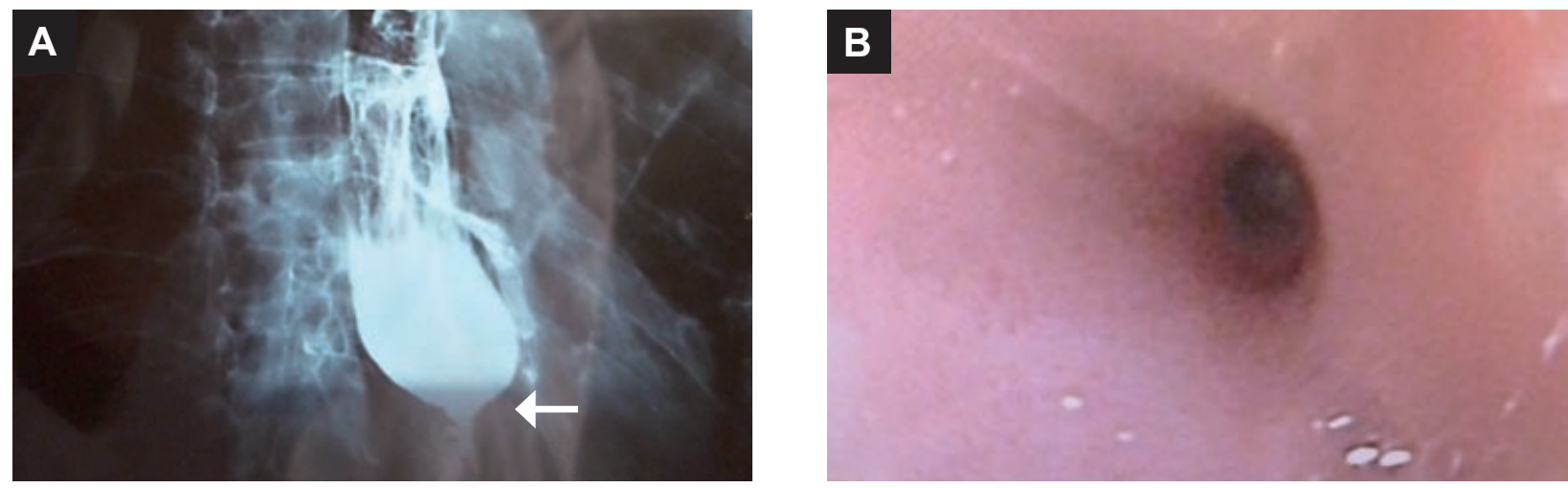

Figura 3. Exámenes auxiliares. A. Esofagograma baritado muestra estrechamiento de la luz en punta de lápiz; B. Endoscopía al mes de tratamiento aún muestra estenosis esofágica concéntrica.

serología para VIH 1, 2 y HTLV-1 fue negativa. El examen coproparasitológico resultó negativo.

En la biopsia de lengua se halló inflamación crónica granulomatosa con células gigantes que fagocitaban células micóticas en gemación, imágenes correspondientes a Paracoccidioides brasiliensis (Figura 1). La biopsia de laringe mostró mucosa de esófago con papilomatosis; coilocitos; moderada hiperplasia de células basales; y linfocitos benignos en el corion; no hubo tejido gástrico ni metaplásico (Figura 2).

Se encontró un patrón con microcalcificaciones bilaterales basales en la radiografía de tórax. El estudio radiológico baritado mostró estrechamiento de la luz esofágica (Figura 3A). La endoscopia confirmó la estenosis a $33 \mathrm{~cm}$ de la boca; la cual no permitió el paso de una guía más delgada pero sí de material de contraste (Figura 3B). No se encontraron alteraciones en la ecografía abdominal de ingreso.

La biopsia endoscópica esofágica señaló tejido escamoso benigno; lámina propia necrótica; resto de tejido con densa infiltración de células inflamatorias mixtas; presencia de granulomas epitelioides, y células gigantes multinucleadas tipo Langhans (Figura 4A). Con la coloración Ziehl-Neelsen se encontró bacilos ácido-alcohol resistentes (Figura 4B). No hubo cambios celulares atípicos. El diagnóstico histológico fue esofagitis granulomatosa por TB con necrosis sin cambios de malignidad.

Debido al antecedente de TB se inició tratamiento antituberculoso estandarizado con kanamicina, pirazinamida, etionamida, levofloxacino, cicloserina y etambutol. Además, recibió itraconazol molido vía oral a razón de 100 mg cada 12 horas, y 100 mg de piridoxina una vez al día. Se realizó una gastrostomía con colocación de tubo de látex a través del cual se continuó el tratamiento. Se monitorizó frecuentemente los valores de gases arteriales, electrolitos, creatinina sérica y audiometría por el uso de medicamentos con potencial ototóxico y neurotóxico, considerando además la disminución de la función renal con la edad y del caso en particular.

El paciente presentó un episodio convulsivo atribuido a un trastorno metabólico (hipoglucemia); no se encontró datos sugerentes de insuficiencia suprarrenal
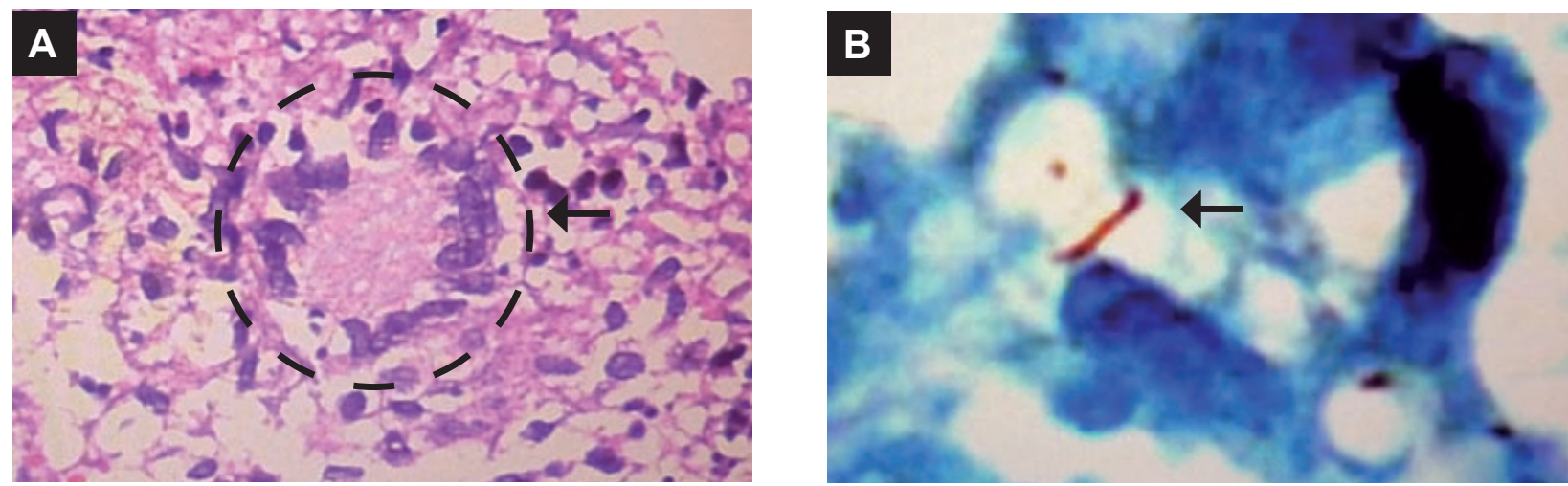

Figura 4. Biopsia de esófago. A. Granulomas epitelioides (coloración HE 400x); B. Bacilos ácido-alcohol resistentes (coloración Ziehl-Neelsen1000x). 
(enfermedad de Addison), dado el riesgo de daño adrenal por TB o por paracoccidiomicosis.

Los síntomas mejoraron rápidamente una vez iniciado el tratamiento antituberculoso. Al cabo de un mes y medio, el paciente fue dado de alta para continuar el tratamiento estandarizado previa coordinación del Comité de Evaluación de Retratamiento Intermedio (CERI) en su centro de salud de procedencia. No se llegó a realizar maniobras de dilatación esofágica.

\section{DISCUSIÓN}

La TB esofágica debe ser considerada dentro del diagnóstico diferencial de disfagia, y no solo en pacientes inmunosuprimidos sino en todos aquellos con antecedentes epidemiológicos de TB o que presentan síntomas esofágicos o mediastínicos sin etiología precisa. El bacilo de Koch es capaz de colonizar prácticamente cualquier órgano.

Se han publicado solo tres casos de tuberculosis esofágica en el Perú: Chávez en 1998; Malca en 2003, y Baños en $2006^{(10)}$. La posibilidad de compromiso extrapulmonar aumenta en los pacientes seropositivos al VIH ${ }^{(5)}$. Tanto la TB como la paracoccidiomicosis se consideran enfermedades oportunistas en la actualidad (11). El tratamiento antituberculoso suele ser inefectivo en estos pacientes requiriendo que la duración sea mayor ${ }^{(1)}$.

El problema de la coinfección por paracoccidiomicosis y tuberculosis ha sido ampliamente reconocido (estimándose entre un 5,5 y 19\% en zonas endémicas); puede presentarse de forma simultánea o secuencial. Las formas similares de presentación clínica y radiológica no permiten una clara distinción entre ambas siendo muchos los casos de paracoccidiomicosis tratados como TB sin tener resultados de baciloscopía positivos ${ }^{(12,13)}$.

Los antimicóticos triazólicos como el itraconazol han mostrado ser eficaces en el tratamiento de la blastomicosis sudamericana ${ }^{(8)}$. Su uso en lugar de anfotericina $\mathrm{B}$ se prefirió en este paciente por el riesgo de nefrotoxicidad añadido. Recientemente, un estudio brasileño encontró resultados semejantes en casos pulmonares al compararlo con voriconazol (14). La cirugía debe reservarse para los casos que no puedan diagnosticarse por endoscopia o biopsia glandular percutánea, o aquellos con complicaciones como fístulas o abscesos que no pueden solucionarse con manejo médico ${ }^{(4,15)}$.
El reporte presenta la limitación de no haber realizado cultivos iniciales para TB ya que la muestra de la endoscopía, al no sospechar de TB esofágica, se colocó en formaldehido. Un cultivo posterior resultó negativo, probablemente por la terapia antituberculosa iniciada. Si bien se atribuye la etiología de la estenosis esofágica a $M$. tuberculosis, otras micobacterias también pueden producirla. Sin embargo, estas requieren condiciones especiales para su cultivo.

Lo particular del caso presentado radica en el uso de tratamiento de segunda línea para TB y la necesidad de recurrir a gastrostomía para administrar la terapia. Asimismo, las dos comorbilidades adicionales en un mismo paciente indican la posibilidad, especialmente en cuadros atípicos, de presentar múltiples complicaciones simultáneas. Aunque no se llegó a determinar la causa de inmunodeficiencia en este paciente, se resalta que existió una coinfección micótica, viral y bacteriana.

\section{AGRADECIMIENTOS}

A los doctores Benjamín Vigo Alcántara y Vicente Maco por su colaboración en la identificación de la biopsia y toma de imágenes. Al Dr. Jaime Vargas por su apoyo en el seguimiento del paciente.

\section{Fuentes de financiamiento}

Autofinanciado.

\section{Conflictos de interés}

Los autores declaran no tener conflictos de interés en la publicación de este artículo.

\section{REFERENCIAS BIBLIOGRÁFICAS}

1. Lozano AS, Leibovich N, Souto G, Sabatini C, Brodersen C, Segal E. Tuberculosis esofágica: presentación de un caso y revisión de la literatura. Acta Gastroenterol Latinoam. 2011;41(1):47-51.

2. Gomes J, Antunes A, Carvalho A, Duarte R. Dysphagia as a manifestation of esophageal tuberculosis: a report of two cases.J Med Case Reports. 2011;5:447.

3. Patnayak R, Reddy MK, Parthasarathy S, Yootla M, Reddy V, Jena A. Unusual presentation of esophageal tuberculosis mimicking malignancy. Saudi J Gastroenterol. 2008;14(2):103-4.

4. Rathinam S, Kanagavel M, Tiruvadanan BS, Santhosam R, Chandramohan SM. Dysphagia due to tuberculosis. Eur J Cardiothorac Surg. 2006;30(6):833-6.

5. Bresky RG, Silva ZN, Madariaga GJ. Esofagitis tuberculosa en un paciente HIV positivo. Rev Med Chil. 2007;135(10):1323-6.

6. Zerpa R, Béjar V, Rojas R. Agentes de infecciones por hongos dimorficos y cryptococcusneoformans. Rev Peru Med Exp Salud Publica. 2011;28(4):685-7. 
7. Shikanai-Yasuda MA, Telles Filho F de Q, Mendes RP, CoIombo AL, Moretti ML. Consenso em paracoccidioidomicose. Rev Soc Bras Med Trop. 2006;39(3):297-310.

8. Burstein Alva Z. Aspectos clínicos de la blastomicosis sudamericana (Paracoccidioidomicosis) en el Perú. Rev Peru Med Exp Salud Publica. 2002;19(1):43-7.

9. Fuchsmann C, Ayari-Khalfallah $\mathrm{S}$, Coulombeau B, Froehlich P. Papilomatosis laríngea. EMC Otorrinolaringología. 2011;40(4):1-8.

10. Baños R, Serrano A, Alberca F, Alajarín M, Albaladejo A. Tuberculosis esofágica. Rev Gastroenterol Perú. 2006;26(2):200-2.

11. Conti Díaz I. A propósito del centenario del descubrimiento de la paracoccidioidomicosis. Principales hitos de la evolución de su conocimiento con especial énfasis en las contribuciones científicas nacionales. Rev Méd Urug. 2010;26(1):45-50.

12. Bertoni T, Takao E, Dias JR. Paracoccidioidomicose e tuberculose: diagnóstico diferencial. J Bras Patole Med Lab. 2010;46(1):17-21.
13. Quagliato Júnior R, Grangeia T de AG, Massucio RA de C, De Capitani EM, Rezende S de M, Balthazar AB. Association between paracoccidioidomycosis and tuberculosis: reality and misdiagnosis. J Bras Pneumol. 2007;33(3):295-300

14. Saievicz de Moraes C, Queiroz-Telles F, Marchiori E, Escuissato DL. Review of lung radiographic findings during treatment of patients with chronic paracoccidioidomycosis. Radiol Bras. 2011;44(1):20-8.

15. Galindo SJ, Tejada GRA. Fístula esofágica por tuberculosis en un pacienteVIH positivo. Reporte de un caso y revisión bibliográfica. Med Int Mex. 2009;25(5):395-8.

Correspondencia: Manuel Montoya Lizárraga Dirección: Av. de la Cultura s/n. Cusco, Perú.

Teléfono: (51) 984931025

Correo electrónico: montoyamanuel@hotmail.com

\section{Consulte las ediciones anteriores de la} Revista Peruana de Medicina Experimental y Salud Pública en

\section{www.scielosp.org}

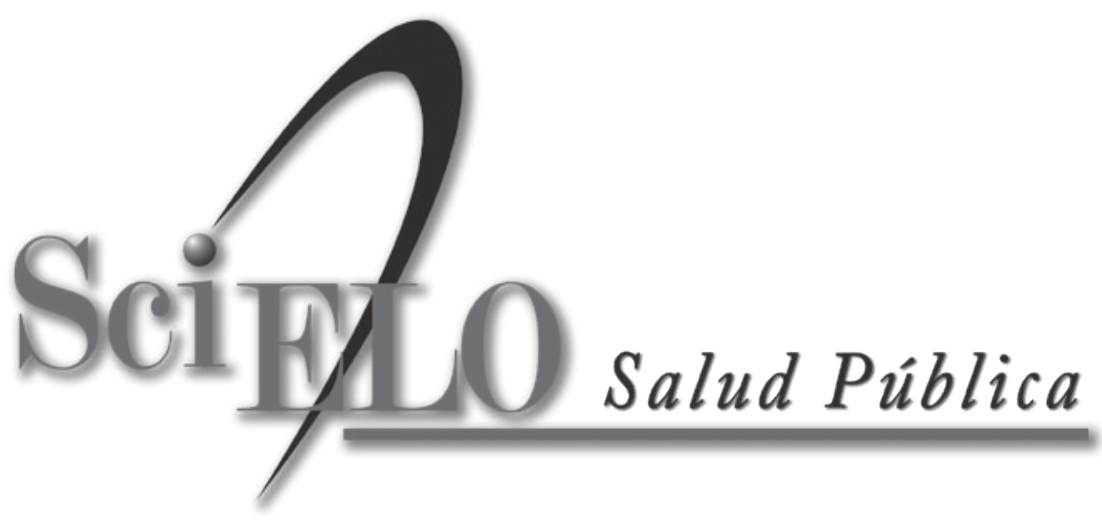

